\title{
EOL-1, the Homolog of the Mammalian Dom3Z, Regulates Olfactory Learning in C. elegans
}

\author{
Yu Shen, ${ }^{1,2}$ Jiangwen Zhang, ${ }^{3}$ John A. Calarco, ${ }^{3}$ and Yun Zhang ${ }^{1,2}$ \\ ${ }^{1}$ Departments of Organismic and Evolutionary Biology, ${ }^{2}$ Center for Brain Science, and ${ }^{3}$ FAS Center for Systems Biology, Harvard University, Cambridge, \\ Massachusetts 02138
}

Learning is an essential function of the nervous system. However, our understanding of molecular underpinnings of learning remains incomplete. Here, we characterize a conserved protein EOL-1 that regulates olfactory learning in Caenorhabditis elegans. A recessive allele of eol-1 (enhanced olfactory learning) learns better to adjust its olfactory preference for bacteria foods and eol-1 acts in the URX sensory neurons to regulate learning. The mammalian homolog of EOL-1, Dom3Z, which regulates quality control of pre-mRNAs, can substitute the function of EOL-1 in learning regulation, demonstrating functional conservation between these homologs. Mutating the residues of Dom3Z that are critical for its enzymatic activity, and the equivalent residues in EOL-1, abolishes the function of these proteins in learning. Together, our results provide insights into the function of EOL-1/Dom $3 \mathrm{Z}$ and suggest that its activity in pre-mRNA quality control is involved in neural plasticity.

Key words: invertebrate olfactory plasticity; molecular underpinnings of learning; pre-mRNA quality control

\section{Introduction}

Learning is critical for survival. Previous studies characterized conserved molecules that regulate learning. For example, PKA (cAMP-dependent protein kinase A) and CREB (cAMPresponsive element binding protein), initially characterized in the long-term facilitation of the gill-withdrawal reflex in Aplysia, play critical roles in olfactory learning in Drosophila and spatial learning in mice (Kandel, 2012). The nematode Caenorhabditis elegans provides an opportunity to further study molecular underpinnings of learning. With a fully sequenced genome encoding $\sim 20,000$ genes, many of which have human homologs (Chalfie and Jorgensen, 1998), C. elegans is highly accessible by genetic approaches. Its small nervous system is well defined (Brenner, 1974; White et al., 1986), allowing characterization of genes in the context of neural circuits. Feeding on bacteria, $C$. elegans uses olfactory sensorimotor circuits to respond to bacterial smells (Bargmann, 2006). Previously, we showed that C. elegans reduces its olfactory preference for pathogenic bacteria,

\footnotetext{
Received Jan. 15, 2014; revised Aug. 19, 2014; accepted Aug. 24, 2014.

Author contributions: Y.S. and Y.Z. designed research; Y.S., J.Z., and J.A.C. performed research; Y.S., J.Z., and J.A.C. analyzed data; Y.S., J.A.C., and Y.Z. wrote the paper.

J.A.C. is supported by an NIH Early Independence Award and Harvard University. Y.Z. is supported by The Esther A. and Joseph Klingenstein Fund, March of Dimes Foundation, The Alfred P. Sloan Foundation, The John Merck Fund and NIH. We thank the Caenorhabditis Genetics Center (funded by NIH Office of Research Infrastructure Programs P40 0D010440) and the C. elegans Gene Knock-out Consortium for strains, the Wellcome Trust Sanger Institute for cosmids, DrC. Daly for whole-genome sequencing, Drs V. Poon, P. McGrath, B. Lesch, A. Boyanov, C. Fang-Yen, and C. Gabel for sharing protocols and programs, Dr S. Sarin for the mouse neuronal RNA library, C. Zhong for assistance in behavioral assays, and $\mathrm{Dr} G$. Harris and other Zhang laboratory members for discussion.

The authors declare no competing financial interests.

Correspondence should be addressed to Dr Yun Zhang, Harvard University, 52 Oxford Street, Northwest Science Building, Cambridge, MA 02138. E-mail: yzhang@oeb.harvard.edu.

J. Zhang's present address: School of Biological Sciences, The University of Hong Kong.

DOI:10.1523/JNEUROSCI.0230-14.2014

Copyright $\odot 2014$ the authors $\quad 0270-6474 / 14 / 3413364-07 \$ 15.00 / 0$
}

such as Pseudomonas aeruginosa strain PA14, after ingesting the bacteria for several hours (Zhang et al., 2005; Ha et al., 2010). We characterized conserved pathways that mediate this form of olfactory learning, including a TGF- $\beta$ pathway and an insulin/ IGF-1 pathway (Zhang and Zhang, 2012; Chen et al., 2013).

Previous studies also characterized inhibitory factors for learning. For example, another CRE-binding protein, CREB2 in Aplysia, inhibits long-term facilitation of the gill-withdrawal reflex by interfering with CREB1-mediated transcription (Bartsch et al., 1995). Inhibiting ATF4, the mammalian homolog of CREB2, in the mouse hippocampus enhances long-term potentiation and spatial memory (Chen et al., 2003). In addition, the Drosophila GABAA receptor Rdl suppresses olfactory associative learning (Liu et al., 2007).

Here, we characterize a new inhibitor of olfactory learning in C. elegans, EOL-1. We report that eol-1 mutants display enhanced olfactory learning. eol-1 encodes a putative protein that has many homologs in eukaryotes, including the mammalian protein Dom3Z implicated in pre-mRNA quality control (Jiao et al., 2013). eol-1 acts in the URX sensory neurons to inhibit learning. Expressing the mouse Dom $3 z$ in eol-1-expressing cells fully rescues the learning phenotype in eol-1 mutants, indicating that EOL-1 shares functional similarities with Dom3Z in regulating learning. Mutating the residues that are critical for the enzymatic activity of Dom 3Z, and the equivalent residues in EOL-1, abolishes the function of these molecules in learning. Together, our findings provide insights into the function of this conserved protein in regulating experience-dependent behavioral plasticity.

\section{Materials and Methods}

Strains and transgenes. C. elegans strains were maintained under standard conditions at $20^{\circ} \mathrm{C}$ (Brenner, 1974). Hermaphrodites were used in the study. The strains that are used include: N2, ZC1279 eol-1(yx29)V, 
A

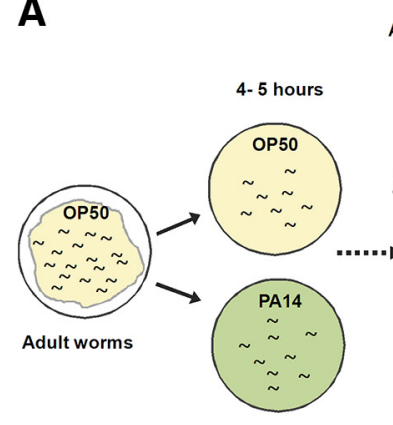

B

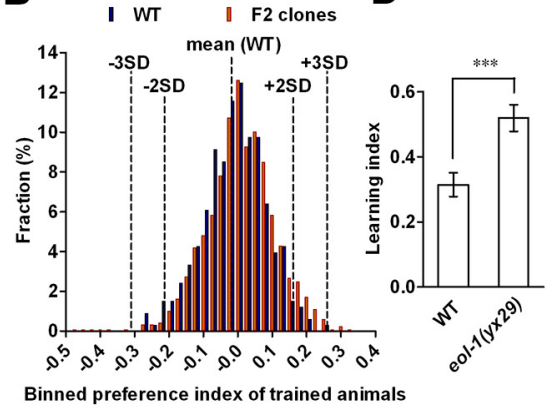

C

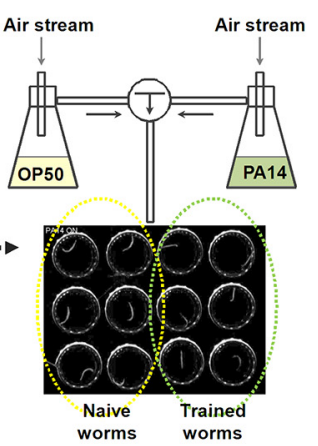

$\mathbf{F}$
$\mathbf{E}$

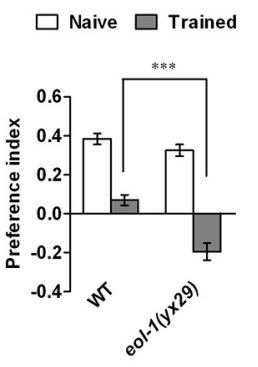

$\square$ Naive $\square$ Trained

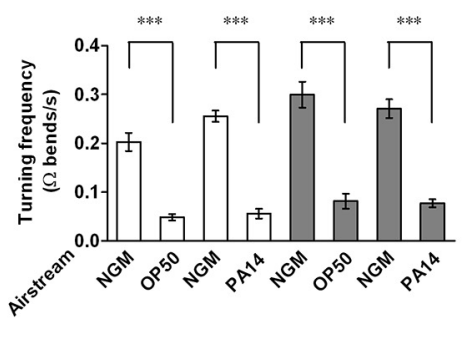

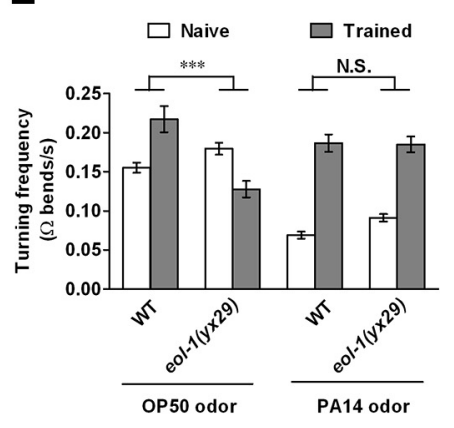

G

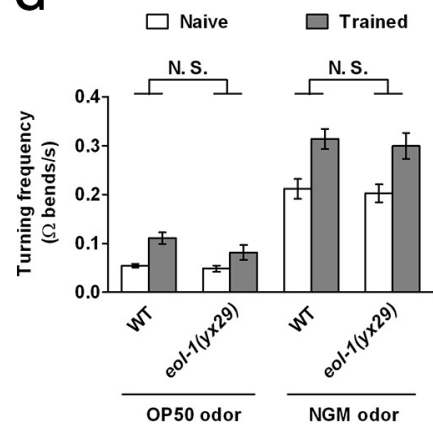

Figure 1. Identification and characterization of the eol-1(yx29) mutant. $A$, Schematics of aversive training with $P$. aeruginosa PA14 and microdroplet assay. $\boldsymbol{B}$, Histogram of binned preference indexes of trained wild type (WT) and F2 clones in the first-round screen. Bin size $=0.025$; columns are slightly nudged for a clear display. SD indicates standard deviation of wild type indexes. $C$, $\boldsymbol{D}$, Preference index $(\boldsymbol{C})$ and learning index $(\boldsymbol{D})$ of wild type and eol-1(yx29) in aversive olfactory learning assay, two-tailed Student's $t$ test; ${ }^{* * *} p<0.001$. $\boldsymbol{E}$, Turning frequency of wild type and eol-1(yx29) in response to the alternating smells of $E$. coli OP50 and $P$. aeruginosa PA14. Two-way ANOVA, a significant genotype $\times$ treatment interaction $\left({ }^{* * *} p<0.001\right)$ was detected for responses to 0 P50 odor (genotype, $p<0.01$; treatment, $p>0.05$ ), but not for responses to PA14 odor (n.S., $p>0.05$ for genotype $\times$ treatment interaction; genotype, $p>0.05$; treatment, $p<0.001$ ). $\boldsymbol{F}$, Turning rate of $e 0 l-1(y \times 29)$ to the alternating smells of NGM buffer and bacteria 0P50 or PA14 (two-tailed Student's test; $\left.{ }^{* * *} p<0.001\right)$. G, WT and eol-1 (yx29) respond similarly to the alternating smells of OP50 and NGM buffer. Two-way ANOVA (n.s., $p>0.05$ for genotype $\times$ treatment interaction; genotype, $p>0.05$; treatment, $p<0.001$ ). C $G, n \geq 9$ assays, mean \pm SEM.

ZC361 lin-15B(n765)X; kyIs30, QZ120 daf-2(e1368)III, CX4998 kyIs140I; nsy-1(ky397)II, FX06514 T26F2.3(tm6514)V(1× outcrossed), ZC1683 eol-1(yx29)V; yxEx858[cosmid T26F2; Punc-122::gfp], ZC1726 eol-1(yx29)V; yxEx891[genomic PCR T26F2.3; Punc-122::gfp], ZC1925 yxEx970[Peol-1::gfp; Punc-122::dsred], ZC1957 yxEx744[Pgcy-36:: mCherry; Punc-122::gfp]; yxEx970[Peol-1::gfp; Punc-122::dsred], ZC2027 eol-1(yx29)V; yxEx1058[Peol-1::eol-1::gfp; Punc-122::gfp], ZC2039 eol1(yx29)V; yxEx1067[Pgcy-36::eol-1::gfp; Punc-122::gfp], ZC2046 wdIs5; yxEx1018[Peol-1::mCherry; Punc-122::gfp], ZC2053 yxEx1068[Pgcy-36:: eol-1::gfp; Punc-122::gfp]; yxEx1018[Peol-1::mCherry; Punc-122::gfp], ZC2124 yxEx1129[Peol-1::eol-1::gfp], ZC2172 yxEx1161[Peol-1:: eol-1RNAi; Punc-122::gfp], ZC2243 eol-1(yx29)V; yxEx1183[Pflp-8:: eol-1::gfp; Punc-122::gfp], CX7102 lin-15(n765)X; qaIs2241 [Pgcy-36::egl1; Pgcy-35::gfp; lin-15(+)]X, ZC2353 eol-1(yx29)V; yxEx1224[Peol-1:: Dom3z.b cDNA; Punc-122::gfp], ZC2456 eol-1(yx29)V; yxEx1268[ Peol-1::eol-1(E185A, D187A)::gfp; Punc-122::gfp], ZC2459 eol-1(yx29)V; yxEx1274[Peol-1::Dom3z.b(E234A, D236A); Punc-122::gfp].

For rescue experiments, cosmids (Wellcome Trust Sanger Institute) were injected into $y \times 29$ at $15 \mathrm{ng} / \mu \mathrm{l}$ and genomic DNAs were injected at $23 \mathrm{ng} / \mu \mathrm{l}(\mathrm{srh}-104)$ or $15 \mathrm{ng} / \mu \mathrm{l}($ eol-1). To generate Peol-1::gfp, Peol-1::eol-1::gfp, Pgcy-36::eol-1::gfp and Pflp-8::eol-1::gfp, the unc-54 $3^{\prime}$ UTR in $P P D 95.77$ was replaced by the eol-1 3' cis-regulatory sequence, a Gateway recombination cassette ( $\mathrm{rfB}$; Invitrogen) was ligated upstream of GFP, and the coding sequence of eol-1 was cloned into the vector to produce the $p P D 95.77-r f B$-eol-1-gfp destination vector. The 908 bp 5' upstream sequence of eol-1 was cloned into $p C R 8$ to generate the entry clone. The mouse Dom $3 z . b$ cDNA was reverse transcribed from the mouse neuronal RNA library. Nucleotide mutations were introduced using QuikChange Site-Directed Mutagenesis Kit (Life Technologies). Cell-specific RNAi constructs were generated as described previously (Esposito et al., 2007). Microinjection (at 10 $\mathrm{ng} / \mu \mathrm{l}$ unless otherwise specified) was performed as described previously (Mello et al., 1991) with either Punc-122::gfp or Punc-122:: dsred as a coinjection marker.
Aversive olfactory learning assay. Control and training plates were prepared by inoculating $10 \mathrm{~cm}$ NGM-agar (nematode growth medium) plates with $0.5 \mathrm{ml}$ overnight NGM culture of E. coli OP50 or P. aeruginosa PA14, respectively, and incubating at $26^{\circ} \mathrm{C}$ for $40-45 \mathrm{~h}$. Adult animals were transferred onto a control plate or a training plate for $4-5 \mathrm{~h}$ at room temperature, washed briefly, placed individually into microdroplets of NGM buffer and subjected to alternating airstreams odorized with overnight culture of OP50 or PA14. The behavioral responses were recorded by a CCD camera at $10 \mathrm{~Hz}$ and analyzed by MATLAB software (MathWorks). The average turning rate (frequency of $\Omega$ bends) of three animals of the identical genotype and treatment in each assay was used to generate the following indexes (the indexes from multiple assays were used for statistical analysis):

$$
\begin{gathered}
I \text { (turning frequency })=\text { number of } \Omega \text { bends/time }(\text { second }) \\
\text { Preference index }=\left(I_{\mathrm{OP} 50}-I_{\mathrm{PA} 14}\right) /\left(I_{\mathrm{OP} 50}+I_{\mathrm{PA} 14}\right)
\end{gathered}
$$

Learning index $=$ preference index (naive)

$$
\text { - preference index (trained). }
$$

Mutagenesis, screen, and mutant identification. Wild-type hermaphrodites at L4 stage (P0) were soaked in $0.5 \%$ ethanemethylsulfonate- (EMS-)M9 solution for $4 \mathrm{~h}$ and then washed five times with $\mathrm{M} 9$ buffer. After recovering for $24 \mathrm{~h}$ on a regular culture plate, $100 \mathrm{EMS}$-treated P0s were transferred to a fresh plate to reproduce. Six-hundred F1 (progeny of P0) animals were isolated and $\sim 2300$ F2 (progeny of F1) were cloned. Mutants that were lethal, sterile, morphologically deformed, or severely uncoordinated in locomotion were excluded; olfactory learning was analyzed in the remaining 1072 F2 clones with the microdroplet assay.

The allele $y \times 29$ was outcrossed four times with an essentially wild-type strain ZC361 and sequenced by Illumina Hi-Seq 2000 (paired-ends, 100 bp read length). Sequencing reads were aligned to the WS220 reference ge- 
A
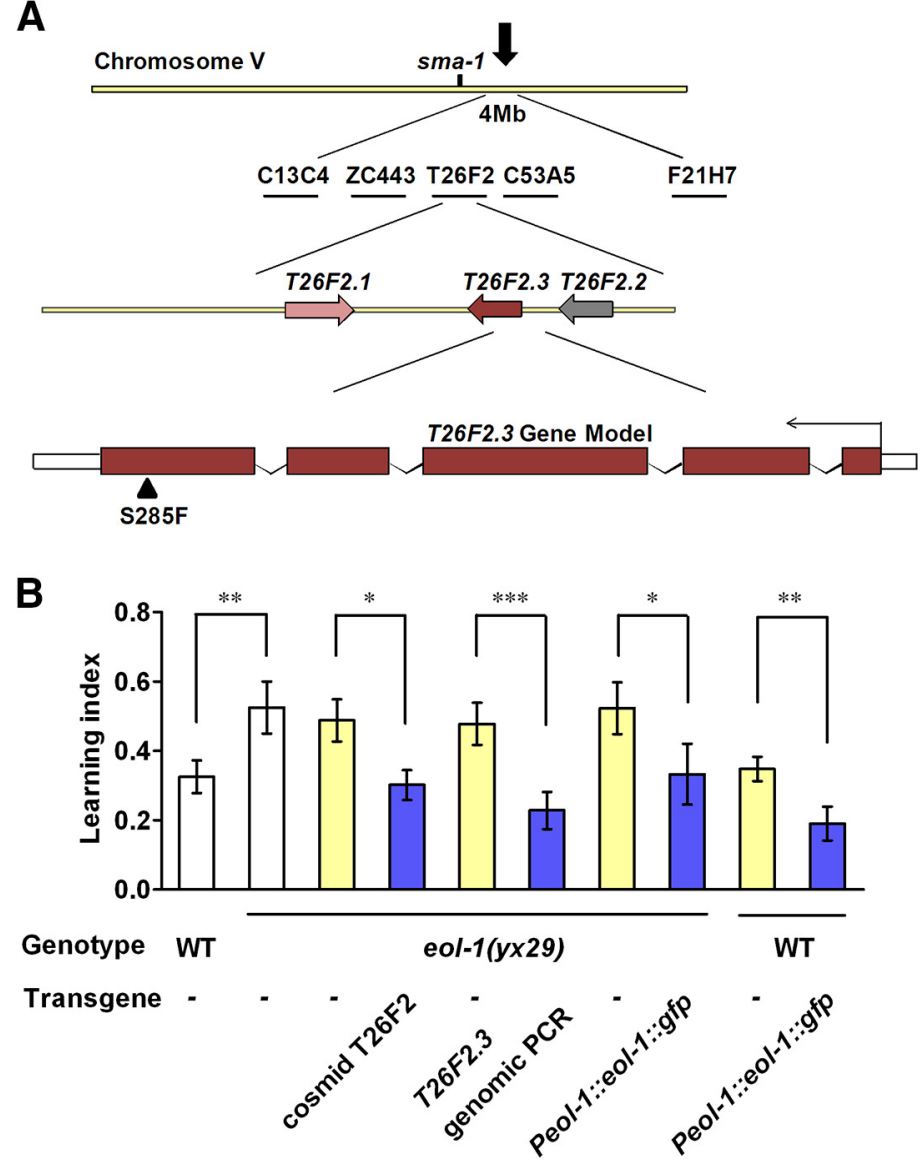

C

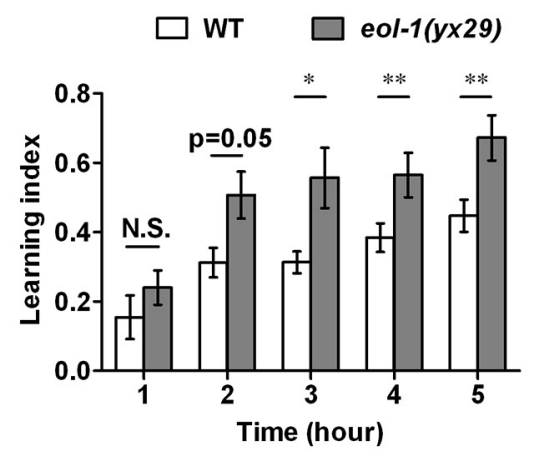

D

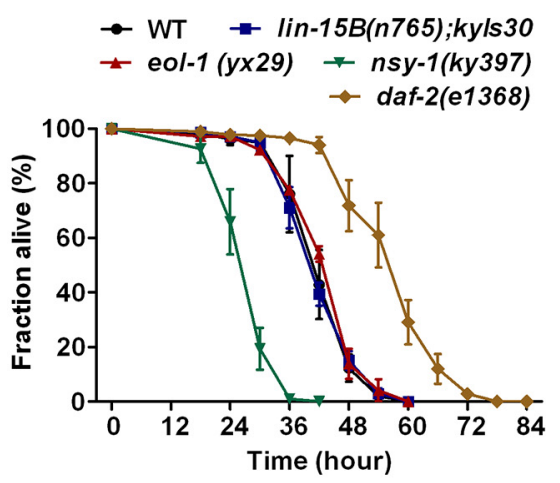

Figure 2. Wild-type T26F2.3 sequence restores normal learning to eol-1(yx29). A, Genomic structure of T26F2.3 sequence (Ensemble Genome Browser, C. elegans WS220). Filled boxes denote exons. Arrowhead denotes the S285F mutation in $y \times 29$. Cosmids tested for rescue are shown. $\boldsymbol{B}$, Expression of $T 26 F 2.3$ restores learning in $y \times 29$. Transgenic animals are compared with nontransgenic siblings, $n \geq 12$ assays each. $C$, Time course of learning. eol-1(yx29) mutants are compared with wild-type controls at each time point, $n \geq 6$ assays each. $\boldsymbol{B}, \boldsymbol{C}$, Two-tailed paired $t$ test; ${ }^{* * *} p<0.001$, ${ }^{* *} p<0.01,{ }^{*} p<0.05$; n.S., not significant, mean \pm SEM. $D$, Slow-killing assays on $P$. aeruginosa PA14; $n=3$ assays each genotype, $n \geq 3$ replicates per assay, log-rank test with Bonferroni correction, no significant difference between eol-1(yx29) and wild type. nsy-1 and daf-2 are controls for reduced and enhanced PA14 resistance, respectively. lin-15B(n765); $k y / s 30$ is a wild-type reporter line used for backcrossing. Error bars indicate SEM.

nome by ELAND and STAMPY; 41 consensus variants were identified in $y \times 29$ using the reads from the parental wild-type and ZC361 as controls.

Microscopy. Fluorescent images were collected with a Nikon Eclipse TE2000-U at $40 \times$ magnification or with an Olympus FV1000A confocal microscope at $20 \times$ or $60 \times$ magnification. Images were processed with Image $(\mathrm{NIH})$.

Slow killing assay. The pathogen resistance to P. aeruginosa PA14 was determined using a slow killing assay similar as described previously (Tan et al., 1999). Briefly, $50 \mu \mathrm{l}$ of overnight Luria Broth culture of PA14 was spread onto a $5 \mathrm{~cm} \mathrm{NGM-agar} \mathrm{plate} \mathrm{and} \mathrm{incubated} \mathrm{at} 37^{\circ} \mathrm{C}$ for $24 \mathrm{~h}$. Fifteen to $20 \mathrm{~L} 4$-stage hermaphrodites were placed on each plate, kept at $25^{\circ} \mathrm{C}$ and scored every $6-7 \mathrm{~h}$. Animals that escaped or died on the wall of the plates were censored.

Food-enhanced butanone chemotaxis assay. As previously described (Torayama et al., 2007), synchronized adults were transferred to E. coli OP50 plates with or without exposure to $10^{-2}$ butanone; after $2 \mathrm{~h}$, animals were tested in standard chemotaxis assays with $10^{-4}$ butanone.

\section{Results}

A high-throughput genetic screen identified a mutant with enhanced olfactory learning

As previously shown, we quantify the preference for the smell of $P$. aeruginosa PA14 over the smell of E. coli OP50 in C. elegans with a microdroplet assay, in which swimming animals are subjected to alternating airstreams odorized with the bacterial cultures (Ha et al., 2010). Swimming C. elegans suppresses sharp body bends ( $\Omega$ bends) in response to attractive olfactory stimuli and increases the bending rate upon the removal of the attractants (Luo et al., 2008). We have shown that short-term training with the pathogenic PA14 induces robust aversive olfactory learning, in which PA14-trained animals reduce their preference for the smell of PA14 (Ha et al., 2010; see Materials and Methods).

To identify novel regulators of olfactory learning, we performed a forward genetic screen with the microdroplet assay to isolate mutants with altered learning (Fig. 1A). Wild-type animals reduce their olfactory preference for PA14 after aversive training; therefore, we screened for mutants that displayed aberrant trained preference index (Fig. 1B). Among 1072 EMSmutagenized F2 clones, we selected $\sim 90$ candidate mutants, whose preference indexes deviated from the wild-type mean by at least 2 standard deviations. By retesting the candidates, we isolated a mutant $y \times 29$. Under the naive condition, $y \times 29$ animals showed wild-type olfactory preference for PA14. However, after $4-5 \mathrm{~h}$ of training, $y \times 29$ displayed a preference index much lower than that of wild type (Fig. $1 C$ ), generating 50\% increase in the learning index (Fig. 1D). $y \times 29$ is a recessive allele, because the progeny of $y \times 29$ crossing with wild type showed wild-type learning (data not shown). Analysis of turning frequency in the microdroplet assay showed that training increased the turning frequency in response to the PA14 smell in $y \times 29$ similarly as in 

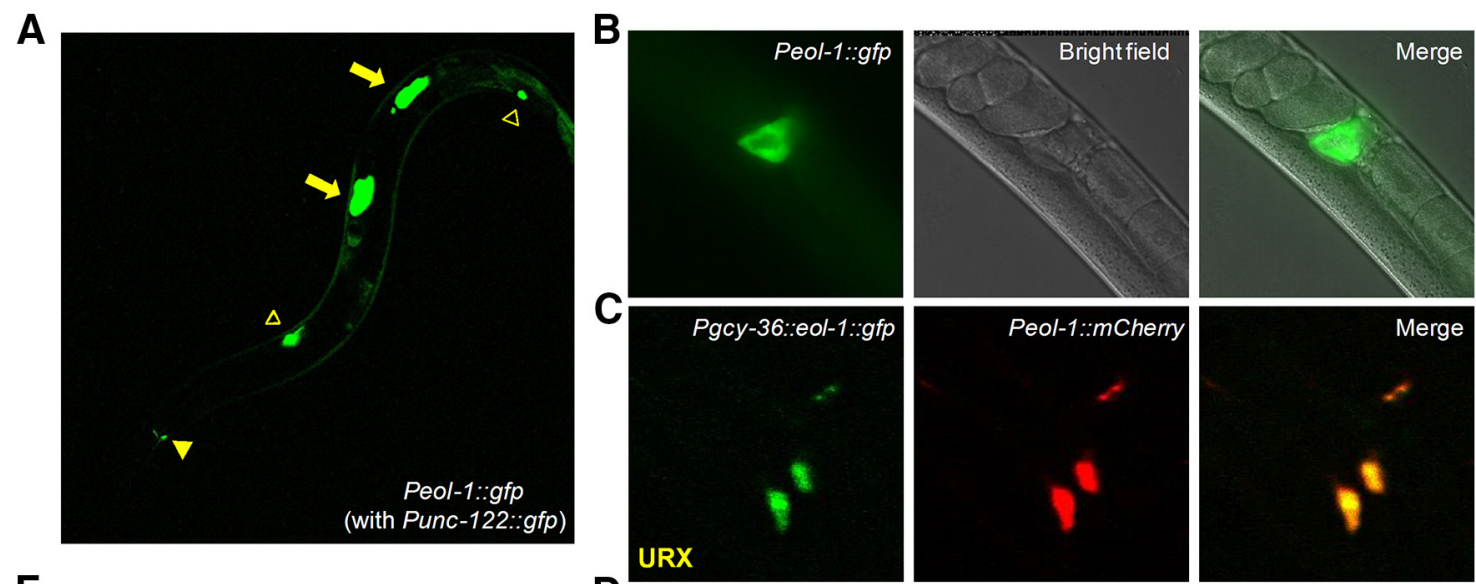

$\mathbf{F}$
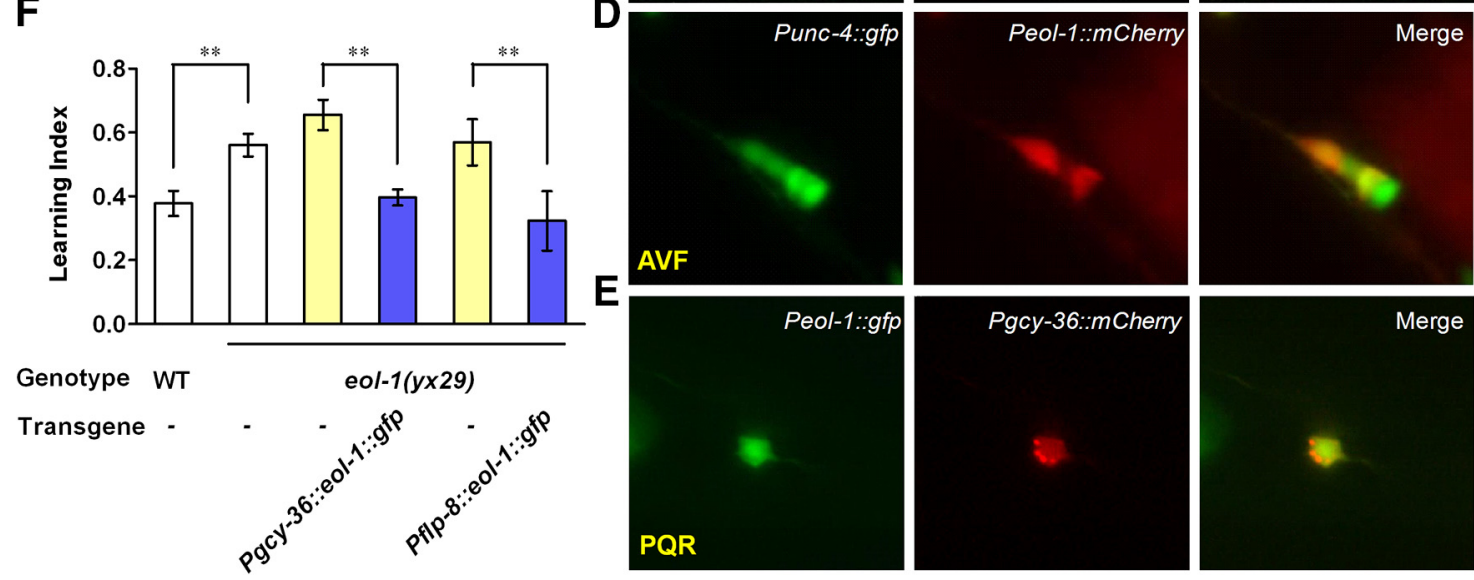

Figure 3. eol-1 is expressed in neurons and functions in URX to regulate olfactory learning. $A$, Expression of Peol-1::gfp in a wild-type adult hermaphrodite. Arrows denote reproductive system and arrowheads denote neurons. The transgenic marker Punc-122:: gfp is expressed in coelomocytes (empty arrowheads). $\boldsymbol{B}-\boldsymbol{E}$, Expression of eol- 1 in hermaphrodite reproductive system (B) and in URX, AVF, and PQR neurons ( $\boldsymbol{C}-\boldsymbol{E})$. The $g c y-36$ promoter drives expression in URX and PQR and the unc-4 promoter drives expression in AVF and SAB. $\boldsymbol{F}$, Aversive olfactory learning of wild type, eol-1(yx29), and transgenic eol-1(yx29) animals that express wild-type eol-1 gene with cell-specific promoters. Transgenic animals are compared with nontransgenic siblings, two-tailed paired Student's $t$ test; ${ }^{* *} p<0.01, n \geq 10$ assays, mean \pm SEM.

wild type and decreased the turning frequency in response to the OP50 smell in $y \times 29$ (Fig. 1E). However, training does not affect the general attraction to bacterial smells in either $y \times 29$ or wild type (Ha et al., 2010; Figure $1 F$ ). In addition, $y \times 29$ and wild type respond similarly to the alternating smells of OP50 and NGM buffer (Fig. 1G). Therefore, the changes in the turning rates to the alternating smells of OP50 and PA14 indicate altered olfactory preference between these bacterial strains. Together, these results show that $y \times 29$, after being trained with the pathogen PA14, reduces its olfactory preference for PA14 over OP50 more than wild type. We named yx29 as eol-1 (enhanced olfactory learning).

\section{The wild-type T26F2.3 sequence restores normal learning to eol-1 (yx29)}

We performed whole-genome sequencing to identify the genetic lesion(s) underlying the learning phenotype of eol-1(yx29). Nonsilent, exonic variants with high confidence were detected in 13 genes on chromosomes $\mathrm{V}$ and $\mathrm{X}$. The functions of these genes were tested by cosmid rescue or by assaying learning in independently generated alleles. Meanwhile, the mutation in eol-1(yx29) was genetically mapped to a $4 \mathrm{Mb}$ region on the right arm of chromosome V (Fig. 2A). Among all the cosmids tested, T26F2 restored wild-type learning to eol-1(yx29) (Fig. 2B). yx29 contains a single-nucleotide mutation in an annotated proteincoding gene T26F2.3. A PCR product containing the wild-type
T26F2.3 coding sequence, a 908 bp 5' cis-regulatory sequence and a $3.1 \mathrm{~kb} \mathrm{3'}$ cis-regulatory sequence rescued the eol-1(yx29) learning phenotype, indicating that the G-to-A transition that changes serine 285 to a phenylalanine in T26F2.3 enhanced learning in eol-1(yx29) (Fig. 2A,B). Expressing a T26F2.3::gfp translational fusion transgene in eol-1 (yx29) also rescued its learning phenotype (Fig. 2B). Thus, T26F2.3 is sufficient to restore normal learning to eol-1(yx29) mutants. Another allele, tm6514, containing 668bp deletion of T26F2.3 also produced an increased learning index $(\mathrm{WT}=0.344 \pm 0.036, \mathrm{tm} 6514=0.484 \pm 0.040$, Student's $t$ test, $p=0.0118, n=24$ assays, mean \pm SEM). In addition, knocking down T26F2.3 by injecting sense and antisense fusion constructs increased learning index (control $=0.216 \pm 0.031$, $\mathrm{RNAi}=0.293 \pm 0.023$, Student's $t$ test, $p=0.0399, n=15$ assays, mean \pm SEM). Together, these results show that T26F2.3 encodes EOL-1. Overexpressing the T26F2.3::gfp fusion transgene in wild type decreased the learning index, suggesting that eol-1 suppresses learning (Fig. 2B).

To examine the temporal profile of the enhanced learning, we measured learning in eol-1 (yx29) and wild type at different time points of training on PA14. We found that eol-1(yx29) learned faster than wild type, with a learning index after $2 \mathrm{~h}$ training comparable to the wild-type learning index induced by $5 \mathrm{~h}$ training (Fig. 2C). The learning indexes of eol-1(yx29) remained higher than wild type throughout the time course, showing $\sim 50 \%$ increase at the end (Fig. $2 C$ ). We also performed slow- 
A M. musculus Dom3Z gi|254939704|ref|NP 291091.2| MEPRGTKRKAEKTEVEKPLHKLPRAVPLLRTQPSLYSGPFPFYRRPSEIG 50 H.sapiens DOM3Z gi|20631980|ref|NP_005501.2| MDPRGTKRGAEKTEVAEPRNKLPRPAPSLPTDPALYSGPFPFYRRPSELG 50 D, melanogaster DXO gi $|45555789|$ ref $\mid$ NP_996492.1| -----MAENAGFISVPWGQHKLG-----------AMYNTPFPSISRPKCIG 35

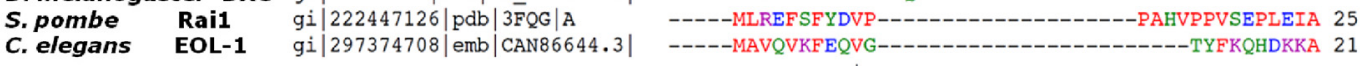

CFSLDAOROYHGDARALRYYSPPPINGPGPDFDLRDGYPDRYOPRDEEVQ ERLDHLLRWVLEHRNOLEGGPG------WLAGATVTWRGHLTKLLTTPYE 144 CFSLDAQROYHGDARALRYYSPPPTNGPGPNFDLRDGYPDRYOPRDEEVQ ERLDHLLCWLLEHRGRLEGGPG-------WLAEAIVTWRGHLTKLITTPYE 144 VCSINASREFVDDASCASYLAGQP--WPPLPFDLNGGIEDVIRKPVENGK RDLEIMLTYIKQHQKELLRQSSSDARNLRLDSDFVTLRGILRQIMCLQYD 133 CYSLSRDRELLLDDSKLSYYYPPP-----LFSDLNTGFPNRFHPPKSD-P DPISIVKDVLMTKG-------------IQMNSSFLTWRGLITKIMCAPLD 106 IPGKLPNR-LNDDPDLYGKLKYP-------LALSLGCKDFKDEGGGDVY ESIFDFTRKTANRGTSLKET---------IDADIFSNRSNLTTIATASKF 103
* * *
: .
$: \quad .: *$ : :

Motif I

RQEGWQLAASRFQGTLYLSEVETPAARAQRLARPPLLRELMYMGYKFEQY MCA-------DKPGGSPDPSGEVNTNVAYCSVLRSRLGNHPLLFSGEVDC 237 RQEGWQLAASRFQGTLYLSEVETPNARAQRLARPPLLRELMYMGYKFEQY MCA-------DKPGSSPDPSGEVNTNVAFCSVLRSRLGSHPLLFSGEVDC 237 NR-SFRVKATLINGNVYMCKEETPEOOLENANMSRAORVMCSWGFKFEOY LTS-_-_---AOAOGKPVTNVPVNEAEEFMGVYRTNLAGILMLYGAELDC 225 PRNHWETYLVMDPTSGIIMMEERTRSETSYANQD----RMCYWGYKFEAI STLPEIWDACSRDQIEQRDNQDVVPDEQYCSIVKINIGKSKLILAGEVDC 202 NREPSEIIALRKNGVIFLYKNEKSYEG-----------KYGGGYNFEKY MTLNEVG---HPHNPNEKVSNATCSKVVLRTSFVSRKETIKVYYASEVDA 188 : . $\quad$ * . $\quad *:: * * \quad$. . . . . . . .

Motif II Motif III LNPOAPCTOPPS------CYVELKTSKEMHSPGOWRSFYR--TDPQAPSTQPPT------CYVELKTSKEMHSPGQWRSFYR------------HKLLKWWAQSFLPGVPNVVAGFRNPDGFVSSLKTFPTMKMFEYVRNDR 319 VDSKEPVDFKDCRVLDSLKFVELKTSVFNMNPHQIRTFKS--------- --FKSANWWSQSFLVGITTLYVGLRDTKGMLQRIDEIDVATLARNKP--- 310 IWDKKPCSAKESDVHSDDGTIEEDASNAENPNLHYVELKTSKKYPLENYG MRKKLLKYWAQSFLLGIGRIIIGFRDDNGILIEMKELFTHQIPKMLRPYF 302 VDKNG------------NFVEMKSTKSDHG--KWLHWNS---------- -----QKHYFOSFLGNVPTIL IGRKDQNDCVYOVDKIKTSEIPKMDVKWK 258

: : * : : Motif IV

--EGWNPSVCMNFCAAFLSFAQSTVVQDDPRLVHLFSWEPGGPVTVSVHR DAPYAFLPSWYVETMTQDLPPLSKTPSPKD 397 --DGWNPSVCMNFCAAFLSFAQSTVVQDDPRLVHLFSWEPGGPVTVSVHQ DAPYAFLPIWYVEAMTODLPSPPKTPSPK- 396 ----WSASAMAWYLEQFLRNLKKLIVNINDPFAVVQVTFLNKHASYEVLR GPEHQILPNWYRDLLKTRS----------- 375 KPNDWTPNRLLVVLEHALEWIKQTVKOHPPSTEFTLSYTGGSKLVLRQII LEHHHHHH--

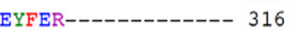

B

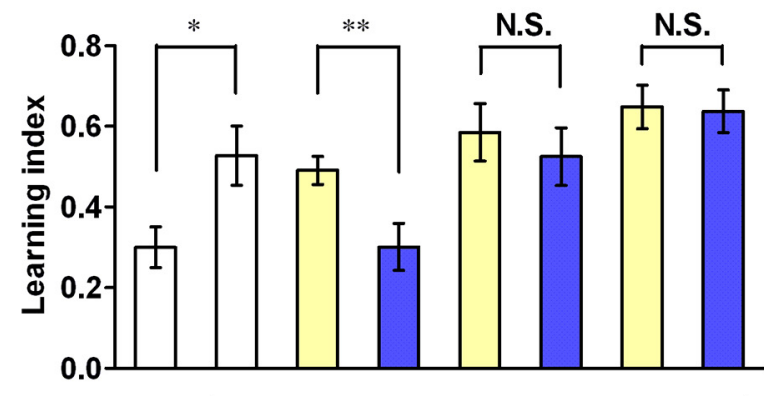

\section{Genotype WT} eol-1(yx29)

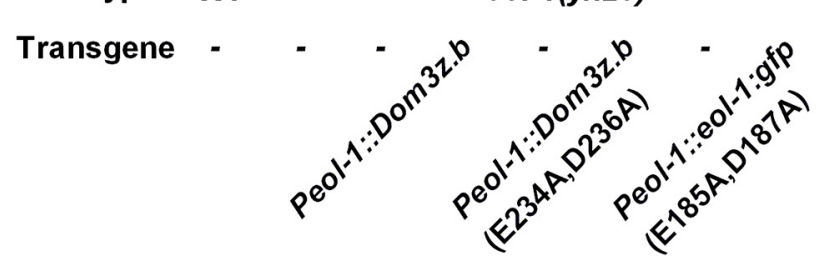

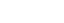

C
M. musculus

H. sapiens

D. melanogaster

S. pombe

C. elegans

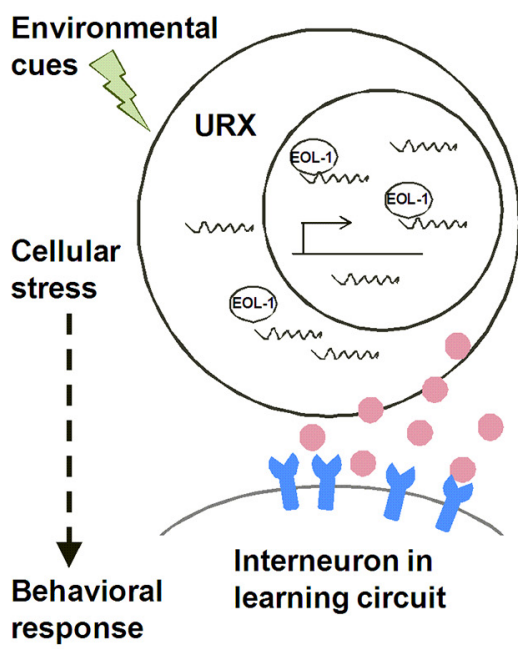

Figure 4. Mouse Dom3z cDNA rescues the learning phenotype of eol-1(yx29). A, Sequence conservation among EOL-1 homologs. *Indicates positions with a single conserved residue; : indicates conservation between groups of strongly similar properties; $\cdot$ indicates conservation between groups of weakly similar properties; and color-code indicates physicochemical properties of amino acids (ClustalW2.1). B, Mouse Dom3z.b CDNA rescues aversive olfactory learning of eol-1(yx29), but the mutated isoform Dom3z.b(E234A, D236A) or eol-1(E185A, D187A) does not. Wild type and eol-1(yx29) are compared using two-tailed Student's $t$ test; $n \geq 4$ assays, ${ }^{*} p<0.05$. Transgenic animals are compared with nontransgenic siblings with two-tailed paired Student's $t$ test; $n=14$ assays, ${ }^{* *} p<0.01$, n.S., not significant, mean \pm SEM. C, Model of EOL-1 function in aversive olfactory learning.

killing assays to measure the resistance to PA14 in $y \times 29$ and found that the survival curve of eol-1 ( $y \times 29)$ was indistinguishable from that of wild type, indicating that eol-1 (yx29) does not learn more because of altered pathogen resistance (Fig. 2D; see Materials and Methods). In addition, using previously established assays (Troemel et al., 1997; Chao et al., 2004), we found that eol-1(yx29) displayed wild-type response to repulsive odors 2-nonanone (chemotaxis index, WT $=-0.927 \pm 0.011$, eol- $1=-0.876 \pm$ 0.020 , Student's $t$ test, $p=0.070, n=4$ trials, $3-4$ replica per trial, mean \pm SEM) and 1 -octanol [response time (second), WT $=$ $1.64 \pm 0.053$, eol $-1=1.63 \pm 0.088$, Student's $t$ test, $p=0.933, n=$ 3 trials, $>20$ animals per trial, mean \pm SEM], indicating a normal olfactory response to repellants. We further tested eol-1(yx29) mutants in another short-term olfactory learning paradigm, food-enhanced butanone chemotaxis, in which animals increase attraction to butanone after exposure to butanone on food (Torayama et al., 2007). We observed no significant difference between eol-1(yx29) and wild type in this assay (learning index, $\mathrm{WT}=0.231 \pm 0.024$, eol $-1=0.242 \pm 0.015, p=0.715$, Student's $t$ test, $n \geq 4$ assays, mean \pm SEM). Therefore, eol- 1 specifically regulates aversive olfactory learning.

\section{eol-1 functions in the URX neuron to regulate olfactory learning}

We characterized the expression pattern of eol-1 by analyzing a transcriptional reporter Peol-1::gfp or Peol-1::mCherry, and a translational reporter Peol-1::eol-1::gfp. These transgenes showed consistent expression patterns, and no difference in eol-1 expres- 
sion was detected between wild type and $y \times 29$. In adult hermaphrodites, Peol-1::gfp was expressed in a few head and tail neurons, and in the reproductive system (Fig. $3 A, B$ ). Using promoters known to drive expression in specific cells, we identified that the head neurons URX and AVF and the tail neuron PQR express eol-1 (Fig. 3C-E).

Next, we used cell-specific promoters to selectively express a gfp-tagged wild-type eol-1 coding sequence in eol-1(yx29). We found that expressing wild-type eol-1::gfp either in the URX, $\mathrm{AQR}$, and PQR neurons using the $g c y$-36 promoter or in the URX and AUA neurons using the $f l p-8$ promoter (Macosko et al., 2009) completely rescued the learning phenotype in eol-1(yx29) (Fig. $3 F$ ). Among the neurons that endogenously express eol-1, only the URX neurons express both $g c y$-36 and $f l p-8$. Killing URX by ectopically expressing a cell death molecule EGL-1 (Chang et al., 2006) significantly decreased the pathogen-induced olfactory learning (learning index, $\mathrm{WT}=0.45 \pm 0.036$; URX-kill $=0.18 \pm$ $0.030, p=1.6 \times 10^{-6}$, Student's $t$ test, $n=18$ assays, mean \pm SEM), confirming the critical role of URX in learning. Together, our results show that EOL-1 acts in the URX sensory neurons to play a negative role in aversive olfactory learning.

\section{The mouse homolog of eol-1, Dom3z, rescues the learning phenotype of eol-1(yx29)}

The EOL-1 protein has several paralogs in C. elegans and homologs in other Caenorhabditis species. EOL-1 is also homologous to Rail and Dox1 in yeast and Dom3Z in mammals (Fig. $4 A$ ). The closest homolog of Dom 3Z in C. elegans is DOM-3. However, the deletion allele dom-3(tm2422) did not show any defect in olfactory learning (data not shown), suggesting the functional specificity of eol-1 in learning.

Because EOL-1 is conserved across species, we evaluated its functional conservation by expressing the cDNA of one isoform of the mouse Dom3z, Dom3z.b, under the eol-1 promoter in eol1(yx29) mutants. Interestingly, the mouse Dom $3 z . b$ cDNA fully rescued the learning phenotype in eol-1(yx29) (Fig. 4B), suggesting that EOL-1 and its mammalian homolog Dom3Z share functional similarities in regulating aversive olfactory learning.

The two yeast homologs of EOL-1, Rail and Dxo1, initiate a quality control system to clear pre-mRNAs with aberrant $5^{\prime}$ caps, which can be produced under normal conditions and during nutritional stress (Jiao et al., 2010; Chang et al., 2012). Similarly, the mammalian homolog Dom3Z plays a central role in degrading aberrantly capped pre-mRNAs (Jiao et al., 2013). To examine whether the enzymatic activity of Dom $3 Z$ is important for learning, we mutated two residues, E234 and D236, which play critical roles in the exonuclease and decapping activities of Dom $3 \mathrm{Z}$ (Chang et al., 2012; Jiao et al., 2013), as well as the equivalent residues in EOL-1, E185 and D187. We found that neither Dom3Z(E234A, D236A) nor EOL-1(E185A, D187A) could rescue the learning phenotype in eol-1(yx29) (Fig. 4B). No difference was detected in localization (data not shown) or fluorescence intensity (WT EOL-1 $=760.14 \pm 20.43$, mutant EOL-1 $=711.17 \pm 25.45, n=50$ transgenic animals each, Student's $t$ test, $p=0.137$, mean \pm SEM) between the EOL-1::GFP fusion and the EOL-1(E185A, D187A)::GFP fusion in these transgenic animals. Thus, the enzymatic activity of Dom $3 Z$ is needed for its function in learning. We also tested whether EOL-1 interacts with the C. elegans exoribonuclease XRN-2, the homolog of the protein partner of yeast Rai1, Rat1 (Stevens and Poole, 1995; Xue et al., 2000; Xiang et al., 2009). However, no complex of EOL-1 and XRN-2 was detected in co-IP assays (data not shown), suggesting that EOL-1 may not interact with XRN-2.

\section{Discussion}

The 5 ' end capping and decapping of eukaryotic mRNA is essential for mRNA synthesis, splicing, export, translation, and stability (Ghosh and Lima, 2010; Topisirovic et al., 2011). Despite the critical role of Dom $3 Z$ in pre-mRNA quality control, the function of Dom3Z in whole organism physiology remains largely unknown.

Here, using genetic screen in C. elegans to identify mutants with altered aversive olfactory learning, we have identified a mutant allele of eol-1, yx29, that displays increased learning (Fig. 1). Although EOL-1 and Dom3Z share weak sequence similarity, expressing a cDNA of Dom $3 z$ in the eol-1-expressing cells fully rescues the learning phenotype in eol-1(yx29) mutants. The four sequence motifs that are shared by Rai1, Dxo 1, and Dom $3 Z$ in the putative active site region (Chang et al., 2012) are also conserved in EOL-1 (Fig. 4A). Strikingly, mutating the residues needed for the enzymatic activity of Dom $3 \mathrm{Z}$ and the equivalent residues in EOL-1 abolishes the function of these proteins in learning. Thus, we propose that aversive training with the pathogenic bacteria $P$. aeruginosa PA14 poses a condition that may increase the amount of abnormally $5^{\prime}$ capped pre-mRNAs in the URX neurons that can be cleared by EOL-1 or Dom3Z. Defective EOL-1 would result in accumulation of aberrantly capped pre-mRNAs, which can serve as a form of cellular stress that generates signals needed for learning (Fig. 4C). Consistently, killing URX strongly reduces learning.

Previously, we mapped an olfactory sensorimotor circuit underlying the aversive olfactory learning induced by pathogenic bacteria (Ha et al., 2010). In this circuit, the interneuron RIA plays an essential role, because animals lacking RIA completely lose the learning ability without compromising their olfactory or immune response to the training pathogen (Ha et al., 2010). The URX neuron, the functional site of EOL-1 in regulating learning, is the major presynaptic neuron of RIA (White et al., 1986), providing a circuit base for URX to signal training effects to RIA to induce learning. The nature of the signal and how it derives from the EOL-1-mediated RNA processing remains to be further investigated.

\section{References}

Bargmann CI (2006) Chemosensation in C. elegans. WormBook 1-29. CrossRef Medline

Bartsch D, Ghirardi M, Skehel PA, Karl KA, Herder SP, Chen M, Bailey CH, Kandel ER (1995) Aplysia CREB2 represses long-term facilitation: relief of repression converts transient facilitation into long-term functional and structural change. Cell 83:979-992. CrossRef Medline

Brenner S (1974) The genetics of Caenorhabditis elegans. Genetics 77:71-94. Medline

Chalfie M, Jorgensen EM (1998) C. elegans neuroscience: genetics to genome. Trends Genet 14:506-512. CrossRef Medline

Chang AJ, Chronis N, Karow DS, Marletta MA, Bargmann CI (2006) A distributed chemosensory circuit for oxygen preference in C. elegans. PLoS Biol 4:e274. CrossRef Medline

Chang JH, Jiao X, Chiba K, Oh C, Martin CE, Kiledjian M, Tong L (2012) Dxol is a new type of eukaryotic enzyme with both decapping and $5^{\prime}-3^{\prime}$ exoribonuclease activity. Nat Struct Mol Biol 19:1011-1017. CrossRef Medline

Chao MY, Komatsu H, Fukuto HS, Dionne HM, Hart AC (2004) Feeding status and serotonin rapidly and reversibly modulate a Caenorhabditis elegans chemosensory circuit. Proc Natl Acad Sci U S A 101:15512-15517. CrossRef Medline

Chen A, Muzzio IA, Malleret G, Bartsch D, Verbitsky M, Pavlidis P, Yonan AL, Vronskaya S, Grody MB, Cepeda I, Gilliam TC, Kandel ER (2003) Inducible enhancement of memory storage and synaptic plasticity in transgenic mice expressing an inhibitor of ATF4 (CREB-2) and C/EBP proteins. Neuron 39:655-669. CrossRef Medline 
Chen Z, Hendricks M, Cornils A, Maier W, Alcedo J, Zhang Y (2013) Two insulin-like peptides antagonistically regulate aversive olfactory learning in C. elegans. Neuron 77:572-585. CrossRef Medline

Esposito G, Di Schiavi E, Bergamasco C, Bazzicalupo P (2007) Efficient and cell specific knock-down of gene function in targeted C. elegans neurons. Gene 395:170-176. CrossRef Medline

Ghosh A, Lima CD (2010) Enzymology of RNA cap synthesis. Wiley Interdiscip Rev RNA 1:152-172. CrossRef Medline

Ha HI, Hendricks M, Shen Y, Gabel CV, Fang-Yen C, Qin Y, Colón-Ramos D, Shen K, Samuel AD, Zhang Y (2010) Functional organization of a neural network for aversive olfactory learning in Caenorhabditis elegans. Neuron 68:1173-1186. CrossRef Medline

Jiao X, Xiang S, Oh C, Martin CE, Tong L, Kiledjian M (2010) Identification of a quality-control mechanism for mRNA $5^{\prime}$-end capping. Nature 467: 608-611. CrossRef Medline

Jiao X, Chang JH, Kilic T, Tong L, Kiledjian M (2013) A mammalian premRNA $5^{\prime}$ end capping quality control mechanism and an unexpected link of capping to pre-mRNA processing. Mol Cell 50:104-115. CrossRef Medline

Kandel ER (2012) The molecular biology of memory: cAMP, PKA, CRE, CREB-1, CREB-2, and CPEB. Mol Brain 5:14. CrossRef Medline

Liu X, Krause WC, Davis RL (2007) GABAA receptor RDL inhibits Drosophila olfactory associative learning. Neuron 56:1090-1102. CrossRef Medline

Luo L, Gabel CV, Ha HI, Zhang Y, Samuel AD (2008) Olfactory behavior of swimming C. elegans analyzed by measuring motile responses to temporal variations of odorants. J Neurophysiol 99:2617-2625. CrossRef Medline

Macosko EZ, Pokala N, Feinberg EH, Chalasani SH, Butcher RA, Clardy J, Bargmann CI (2009) A hub-and-spoke circuit drives pheromone attraction and social behaviour in C. elegans. Nature 458:1171-1175. CrossRef Medline

Mello CC, Kramer JM, Stinchcomb D, Ambros V (1991) Efficient gene transfer in C.elegans: extrachromosomal maintenance and integration of transforming sequences. EMBO J 10:3959-3970. Medline

Stevens A, Poole TL (1995) 5'-Exonuclease-2 of saccharomyces cerevisiae. Purification and features of ribonuclease activity with comparison to $5^{\prime}$ exonuclease-1. J Biol Chem 270:16063-16069. CrossRef Medline

Tan MW, Mahajan-Miklos S, Ausubel FM (1999) Killing of Caenorhabditis elegans by pseudomonas aeruginosa used to model mammalian bacterial pathogenesis. Proc Natl Acad Sci U S A 96:715-720. CrossRef Medline

Topisirovic I, Svitkin YV, Sonenberg N, Shatkin AJ (2011) Cap and capbinding proteins in the control of gene expression. Wiley Interdiscip Rev RNA 2:277-298. CrossRef Medline

Torayama I, Ishihara T, Katsura I (2007) Caenorhabditis elegans integrates the signals of butanone and food to enhance chemotaxis to butanone. J Neurosci 27:741-750. CrossRef Medline

Troemel ER, Kimmel BE, Bargmann CI (1997) Reprogramming chemotaxis responses: sensory neurons define olfactory preferences in C. elegans. Cell 91:161-169. CrossRef Medline

White JG, Southgate E, Thomson JN, Brenner S (1986) The structure of the nervous system of the nematode Caenorhabditis elegans. Philos Trans R Soc Lond B Biol Sci 314:1-340. CrossRef Medline

Xiang S, Cooper-Morgan A, Jiao X, Kiledjian M, Manley JL, Tong L (2009) Structure and function of the $5^{\prime}->3^{\prime}$ exoribonuclease Rat 1 and its activating partner Rail. Nature 458:784-788. CrossRef Medline

Xue Y, Bai X, Lee I, Kallstrom G, Ho J, Brown J, Stevens A, Johnson AW (2000) Saccharomyces cerevisiae RAI1 (YGL246c) is homologous to human DOM3Z and encodes a protein that binds the nuclear exoribonuclease Rat1p. Mol Cell Biol 20:4006-4015. CrossRef Medline

Zhang X, Zhang Y (2012) DBL-1, a TGF-beta, is essential for Caenorhabditis elegans aversive olfactory learning. Proc Natl Acad Sci U S A 109:1708117086. CrossRef Medline

Zhang Y, Lu H, Bargmann CI (2005) Pathogenic bacteria induce aversive olfactory learning in Caenorhabditis elegans. Nature 438:179-184. CrossRef Medline 